\title{
MODULES OVER POLYDISC ALGEBRAS
}

\author{
BY \\ WALTER RUDIN( ${ }^{(1)}$ AND E. L. STOUT( $\left.{ }^{2}\right)$
}

Introduction. We shall be concerned with certain analytic covers of the polydisc $U^{N}=\left\{\left(z_{1}, \ldots, z_{N}\right):\left|z_{1}\right|<1, \ldots,\left|z_{N}\right|<1\right\}$ in complex $N$-space, $C^{N}$. We will consider a collection $\mathscr{K}_{N}$ of objects $\Delta: \Delta \in \mathscr{K}_{N}$ if $\Delta$ is an open subset of a Stein manifold and if there is a neighborhood $\Omega$ of $\bar{\Delta}$ and a proper holomorphic map $\Phi$ from $\Omega$ onto a neighborhood of $\bar{U}^{N}$ which satisfies

(1) $\Delta=\Phi^{-1}\left(U^{N}\right)$, and

(2) $\Phi$ is a local homeomorphism at each point of $\Phi^{-1}\left(T^{N}\right)$.

Here $T^{N}=\left\{\left(z_{1}, \ldots, z_{N}\right):\left|z_{1}\right|=\cdots=\left|z_{N}\right|=1\right\}$, the distinguished boundary of $U^{N}$. It follows from the definition of "proper" (compact sets have compact inverse images) that $\bar{\Delta}$ is compact.

Given a $\Delta \in \mathscr{K}_{N}$ and an associated $\Phi$, the triple $\left(\Delta, \Phi \mid \Delta, U^{N}\right)$ is an analytic cover, in the sense of [5]. We will make frequent use of the properties of such covers, often without explicit reference. One of their important properties is that $\Phi$ has a well-defined multiplicity: there is an integer $\lambda$ and an analytic variety $V$ in $U^{N}(\operatorname{dim} V<N)$ such that each point of $U^{N} \backslash V$ has exactly $\lambda$ preimages in $\Omega$.

We will study two algebras naturally associated with $\Delta$,

$$
A(\Delta)=\{f \in C(\bar{\Delta}): f \text { is holomorphic in } \Delta\}
$$

and

$$
H^{\infty}(\Delta)=\{f: f \text { is bounded and holomorphic in } \Delta\} .
$$

These algebras are modules over their subalgebras

$$
\Phi^{*} A\left(U^{N}\right)=\left\{f \circ \Phi: f \in A\left(U^{N}\right)\right\}
$$

and

$$
\Phi^{*} H^{\infty}\left(U^{N}\right)=\left\{f \circ \Phi: f \in H^{\infty}\left(U^{N}\right)\right\} .
$$

In $\S \mathrm{I}$ we show that these modules are actually free, and that their rank equals the multiplicity of $\Phi$ (Theorem I.4). Certain analytic consequences of this are also developed in $\S$ I. An example shows that the above result can fail for maps $\Phi$ which violate condition (2).

In the one-dimensional case, results of this kind have been obtained by Alling. The elements of $\mathscr{K}_{1}$ are simply the Riemann surfaces considered in [1].

Received by the editors April 15, 1968 and, in revised form May 24, 1968.

(1) Partially supported by NSF Grant GP-6764.

(2) Research Associate of the Office of Naval Research. 
Given $\Delta^{\prime}, \Delta \in \mathscr{K}_{N}$ and an associated $\Phi: \Delta \rightarrow U^{N}$, we write $\Psi \in \mathscr{M}\left(\Delta^{\prime}, \Delta\right)$ if $\Psi$ is a proper holomorphic map of a neighborhood of $\bar{\Delta}^{\prime}$ onto a neighborhood of $\bar{\Delta}$ which is locally a homeomorphism at each point of $\Psi^{-1}\left(\Phi^{-1}\left(T^{N}\right)\right)$ and which satisfies $\Delta^{\prime}=\Psi^{-1}(\Delta)$. The set $\mathscr{M}\left(\Delta^{\prime}, \Delta\right)$ depends only on $\Delta$ and $\Delta^{\prime}$ and not on $\Phi$, for, as we shall see, the set $\Phi^{-1}\left(T^{N}\right)$ is independent of $\Phi$.

If $\Psi \in \mathscr{M}\left(\Delta^{\prime}, \Delta\right)$, then $A\left(\Delta^{\prime}\right)$ and $H^{\infty}\left(\Delta^{\prime}\right)$ are again modules over $\Psi^{*} A(\Delta)$ and $\Psi^{*} H^{\infty}(\Delta)$ respectively. This module structure will be studied in a subsequent paper.

$\S$ II deals with the case in which both $\Delta$ and $\Delta^{\prime}$ are polydiscs. The special form of proper holomorphic maps from $U^{k}$ to $U^{N}$ is discussed. Combining this with the results of $\S \mathrm{I}$, the following extension theorem is obtained:

THEOREM. Let $\Phi$ be a biholomorphic map of $U^{k}$ onto a closed analytic submanifold $V$ of $U^{N}$. If $\Phi$ is holomorphic and one-to-one in a neighborhood of $\bar{U}^{k}$, then there is a bounded linear operator $E: H^{\infty}\left(U^{k}\right) \rightarrow H^{\infty}\left(U^{N}\right)$ which maps $A\left(U^{k}\right)$ into $A\left(U^{N}\right)$, and which extends functions on $V$ to functions in $U^{N}$ in the sense that

$$
(E f) \circ \Phi=f
$$

for every $f \in H^{\infty}\left(U^{k}\right)$.

An example shows that such an extension need not exist if $\Phi$ behaves badly near the boundary.

In §III, which is independent of the preceding ones, we obtain a rather general theorem on extending bounded holomorphic functions from an element of $\mathscr{K}_{1}$ embedded in a $U^{M}$ to bounded holomorphic functions in $U^{M}$. On the one hand, this is a generalization of a previous result [13], and on the other, it is a model of what we would like to prove about extending functions from a $\Delta \in \mathscr{K}_{N}$ which is embedded in a $U^{M}$.

Certain notations will be used consistently. If $\mathfrak{M}$ is a complex manifold, and if $z \in \mathfrak{M}$, then $\mathcal{O}(\mathfrak{M}), \mathcal{O}_{\mathfrak{M}}$, and $\mathcal{O}_{z}$ will denote, respectively, the algebra of all holomorphic functions on $\mathfrak{M}$, the sheaf of germs of such functions, and the stalk of this sheaf at $z$. A holomorphic map $\Phi: \mathfrak{M} \rightarrow \mathfrak{R}$, where $\mathfrak{M}$ and $\mathfrak{R}$ are complex manifolds of dimension $m$ and $n$, is said to be nonsingular at $z \in \mathfrak{M}$ if there exist neighborhoods $V$ of $z$ and $W$ of $\Phi(z)$ and biholomorphic maps $\alpha$ and $\beta$ of $V$ and $W$ onto open subsets of $C^{m}$ and $C^{n}$, respectively, such that $\beta \circ \Phi \circ \alpha^{-1}$ is nonsingular in the sense of [5; p. 16]. If $\Phi$ is nonsingular at each point of its domain, we say simply that $\Phi$ is nonsingular.

I. Analytic covers of polydiscs. We begin this section with some general properties of elements of $\mathscr{M}\left(\Delta^{\prime}, \Delta\right)$ and then apply these to the special case that $\Delta$ is a polydisc.

In the definition of the elements $\Delta$ of $\mathscr{K}_{N}$ we required the existence of a neighborhood $\Omega$ of $\bar{\Delta}$ and a proper holomorphic map $\Phi$ from $\Omega$ to a neighborhood of $\bar{U}^{N}$ in $C^{N}$; this $\Phi$ is to be a local homeomorphism at each point of $\Phi^{-1}\left(T^{N}\right)$. It is 
important to observe that the set $\Phi^{-1}\left(T^{N}\right)$ can be described in terms of the algebra $A(\Delta)$; thus $\Phi^{-1}\left(T^{N}\right)$ does not depend on the particular choice of $\Phi$ :

\section{I.1. LEMma $\Phi^{-1}\left(T^{N}\right)$ is the Shilov boundary for $A(\Delta)$.}

Proof. Let $B=\Phi^{-1}\left(T^{N}\right)$. We claim that every $z \in B$ is a peak point for some element of $A(\Delta)$. If $z \in B$, there exists $f \in A\left(U^{N}\right)$ such that $f(\Phi(z))=1$ but $|f(\zeta)|<1$ at every other point $\zeta \in \bar{U}^{N}$. Since $A(\Delta)$ separates points on $\bar{\Delta}$, there exists $g \in A(\Delta)$ such that $g(z)=1$ but $g(w)=0$ at every other point $w \in \Phi^{-1}(\Phi(z))$. If $V$ is a neighborhood (in $\bar{\Delta}$ ) of $z$, if $M$ is a sufficiently large positive integer, and if $h=(f \circ \Phi)^{M} g$, then $h(z)=1,|h|<9 / 8$ on $\bar{\Delta}$, and $|h|<1 / 8$ off $V$. Thus Bishop's characterization of peak points [2, Theorem 2] shows that $z$ is a peak point for $A(\Delta)$. Hence $B$ is a subset of the Shilov boundary.

To prove that $B$ is a boundary for $A(\Delta)$ we must show that $|f|$ attains its maximum on $B$ if $f \in A(\Delta)$. To do this, suppose $0<t<1$, let $T_{t}^{N}$ be the distinguished boundary of the polydisc $U_{t}^{N}\left(z \in U_{t}^{N}\right.$ if and only if $\left.t^{-1} z \in U^{N}\right)$, and put $\Delta_{t}=\Phi^{-1}\left(U_{t}^{N}\right)$. If $f \in A(\Delta)$, then $f \in \mathcal{O}\left(\bar{\Delta}_{t}\right)$, so [6] implies that there is $z_{t} \in \Phi^{-1}\left(T_{t}^{N}\right)$ such that $\left|f\left(z_{t}\right)\right|=\sup \left\{|f(z)|: z \in \Delta_{t}\right\}$. The set $\left\{z_{t}: 0<t<1\right\}$ has a limit point $z_{1} \in B$, and $|f|$ attains its maximum at $z_{1}$. This completes the lemma.

Now suppose $\Delta^{\prime}, \Delta \in \mathscr{K}_{N}, \Phi \in \mathscr{M}\left(\Delta^{\prime}, \Delta\right), \Omega^{\prime}$ is a neighborhood of $\bar{\Delta}^{\prime}$ which $\Phi$ maps properly onto a neighborhood $\Omega$ of $\bar{\Delta}$, and $\Omega^{\prime}=\Phi^{-1}(\Omega)$. The mapping $\Phi$ gives rise to a sheaf $\mathscr{S}$ on $\Omega$ (called the direct image sheaf of $\mathcal{O}_{\Omega^{\prime}}$ ), in the following manner: if $V$ is an open set in $\Omega$, the sections of $\mathscr{S}$ over $V$ are the holomorphic functions on $\Phi^{-1}(V)$. The set $\Gamma(V, \mathscr{S})=\mathcal{O}\left(\Phi^{-1}(V)\right)$ of these sections may be regarded as an $\mathcal{O}(V)$-module: If $f \in \mathcal{O}(V)$ and $g \in \Gamma(V, \mathscr{S})$, define $f g$ to be the element $(f \circ \Phi) g$ of $\Gamma(V, \mathscr{S})$.

Since the fibers $\Phi^{-1}(\Phi(z))$ are finite, [11, Theorem 7, p. 81] (which as the referee has pointed out to us, was proved by Oka [16]) shows that $\mathscr{S}$ is a coherent analytic sheaf. For our purposes, it is necessary to know somewhat more:

I.2. Lemma 2. If $\Phi$ has multiplicity $\lambda$, then $\mathscr{S}$ is a locally free sheaf of rank $\lambda$ over $\mathcal{O}_{\Omega}$.

Proof. Explicitly, the assertion is that every $z \in \Omega$ has a neighborhood $V$ such that $\mathscr{S} \mid V$ is isomorphic to the direct sum of $\lambda$ copies of $\mathcal{O}_{V}$. Since $\mathscr{S}$ is coherent, it is enough to prove that every stalk $\mathscr{S}_{z}(z \in \Omega)$ is isomorphic (as an $\mathcal{O}_{z}$-module) to the direct sum of $\lambda$ copies of $\mathcal{O}_{z}$.

Indeed, let us assume that this last statement has been proved. For a fixed $z \in \Omega$, let $\left(s_{1}\right)_{z}, \ldots,\left(s_{\lambda}\right)_{z} \in \mathscr{S}_{z}$ be germs that constitute a free basis of $\mathscr{S}_{z}$ over $\mathcal{O}_{z}$. Since $\mathscr{S}$ is coherent, $z$ has a neighborhood $V_{1}$ such that $\left(s_{1}\right)_{\zeta}, \ldots,\left(s_{\lambda}\right)_{\zeta}$ generate $\mathscr{S}_{\zeta}$ for every $\zeta \in V_{1}$. Let $\mathscr{R}$ be the sheaf of relations of the sections $s_{1}, \ldots, s_{\lambda} \in \Gamma\left(V_{1}, \mathscr{S}\right)$. The stalk $\mathscr{R}_{z}$ is the zero module since $\left(s_{1}\right)_{z}, \ldots,\left(s_{\lambda}\right)_{z}$ are free generators of $\mathscr{S}_{z}$; since $\mathscr{R}$ is itself coherent it follows that 0 generates the stalks $\mathscr{R}_{\zeta}$ for all $\zeta$ in some neighborhood $V$ of $z, V \subset V_{1}$. So $\mathscr{R}=0$ in $V$, which says that $\mathscr{S} \mid V$ is isomorphic to $\left(\mathcal{O}_{V}\right)^{\lambda}$. 
We now consider two cases. The first is that in which $\Phi^{-1}(z)$ consists of $\lambda$ distinct points, say $w_{1}, \ldots, w_{\lambda}$. Then there is a neighborhood $V$ of $z$ and there are pairwise disjoint neighborhoods $W_{i}$ of $w_{\imath}$ such that $\Phi$ maps each $W_{i}$ biholomorphically onto $V$. Let $\psi_{i}: V \rightarrow W_{i}$ be inverse to $\Phi$. Every germ $s_{z} \in \mathscr{S}_{z}$ is represented by a $\lambda$-tuple of functions $\left(f_{1}, \ldots, f_{\lambda}\right)$, where each $f_{i}$ is holomorphic in some neighborhood of $w_{i}$. The map $s_{z} \rightarrow\left(\tilde{f}_{1}, \ldots, \tilde{f}_{\lambda}\right)$, where $\tilde{f}_{i}$ is the germ at $z$ of the function $f_{i} \circ \psi_{i}$, is an isomorphism of $\mathscr{S}_{z}$ with $\left(\mathcal{O}_{z}\right)^{\lambda}$.

The case in which $\Phi^{-1}(z)$ consists of fewer than $\lambda$ points is not quite so casy. Fix one $w \in \Phi^{-1}(z)$ and let $\mu$ be the branching order of $\Phi$ at $w$ [5, p. 103]. Let $H \subset \mathcal{O}_{\Omega^{\prime}, \text { w }}$ consist of the germs at $w$ of the functions $g \circ \Phi$, where $g$ is holomorphic near $z$. Since $H$ is isomorphic to $\mathcal{O}_{\Omega, \mathrm{z}}$, we have to show that $\mathcal{O}_{\Omega^{\prime}, \mathrm{w}}$ is a free module over $H$, of rank $\mu$.

Let $\tilde{H}$ and $\tilde{\mathcal{O}}_{\Omega^{\prime} \text {, w }}$ be the quotient fields of $H$ and $\mathcal{O}_{\Omega^{\prime}, \mathrm{w}}$. We claim that

(i) $\mathcal{O}_{\Omega^{\prime}, \text { w }}$ is the integral closure of $H$ in $\tilde{\mathcal{O}}_{\Omega^{\prime}, w}$, and

(ii) $\tilde{\mathcal{O}}_{\Omega^{\prime}, \mathrm{w}}$ is a finite algebraic extension of $\tilde{H}$.

Since $\mathcal{O}_{\Omega, \mathrm{z}}$ and $\mathcal{O}_{\Omega^{\prime}, \mathrm{w}}$ (hence also $H$ ) are unique factorization domains [5, p. 72] they are integrally closed $\left[14\right.$, p. 261] and therefore (i) and (ii) will imply that $\mathcal{O}_{\Omega^{\prime}, \mathrm{w}}$ is a finite $H$-module [14, p. 265, Corollary 1$]\left({ }^{3}\right)$. But since $\mathcal{O}_{\Omega, \mathrm{z}}$ and $\mathcal{O}_{\Omega^{\prime}, \text { w }}$ are just the rings of convergent power series in $N$ complex variables, it then follows from [4, Korollar 5] that $\mathcal{O}_{\Omega^{\prime}, \text { w }}$ is actually free over $H$. The rank of $\mathcal{O}_{\Omega^{\prime}, \text { w }}$ over $H$ must then clearly be equal to the branching order of $\Phi$ at $w$, and this is the desired conclusion.

We turn to the proof of (i) and (ii). The point $w$ has a neighborhood basis $\left\{V_{\alpha}\right\}$ such that $\left(V_{\alpha}, \Phi \mid V_{\alpha}, \Phi\left(V_{\alpha}\right)\right)$ is an analytic cover of multiplicity $\mu$. Hence [5, p. 104] every $f \in \mathcal{O}_{\Omega^{\prime}, \text { w }}$ satisfies a monic polynomial equation

$$
f^{\mu}+h_{\mu-1} f^{\mu-1}+\cdots+h_{0}=0 \quad\left(h_{i} \in H\right) .
$$

Thus $\mathcal{O}_{\Omega^{\prime}, \mathrm{w}}$ is a subset of the integral closure of $H$ in $\tilde{\mathcal{O}}_{\Omega^{\prime}, \mathrm{w}}$. On the other hand, every $x \in \tilde{\mathcal{O}}_{\Omega^{\prime}, \mathrm{w}}$ which is integral over $H$ is also (trivially) integral over the larger ring $\mathcal{O}_{\Omega^{\prime}, \mathrm{w}}$, and since $\mathcal{O}_{\Omega^{\prime}, \mathrm{w}}$ is integrally closed (as noted above), it follows that $x \in \mathcal{O}_{\Omega^{\prime}, \mathrm{w}}$. Hence (i) is true.

Since (1) holds for every $f \in \mathcal{O}_{\Omega^{\prime}, \mathrm{w}}$, the usual proof of the fact that the algebraic numbers form a field shows that every $x \in \tilde{\mathcal{O}}_{\Omega^{\prime}, \mathrm{w}}$ satisfies an equation

$$
a_{m} x^{m}+a_{m-1} x^{m-1}+\cdots+a_{0}=0 \quad\left(a_{i} \in H, a_{m} \neq 0\right) .
$$

Multiplying this by $a_{m}^{m-1}$, we see that $a_{m} x$ is integral over $H$. By (i), $a_{m} x \in \mathcal{O}_{\Omega^{\prime}, \mathrm{w}}$, and hence $f=a_{m} x$ satisfies an equation of the form (1). In other words, every $x \in \tilde{\mathcal{O}}_{\Omega^{\prime}, \mathrm{w}}$ is algebraic over $H$, of degree $\leqq \mu$. Pick $x_{0} \in \tilde{\mathcal{O}}$ so that its degree over $H$ is maximal. If there were an $x_{1} \in \tilde{\mathcal{O}}_{\Omega^{\prime}, \mathrm{w}}, x_{1} \notin \tilde{\mathcal{O}}_{\Omega^{\prime} \text {, w }}\left(x_{0}\right)$, then the dimension of the field $\tilde{H}\left(x_{0}, x_{1}\right)$ would be larger than that of $\tilde{H}\left(x_{0}\right)$ (as vector spaces over $\tilde{H}$ ). The

$\left.{ }^{3}\right)$ As the referee has pointed out, the fact that $\mathcal{O}_{\Omega^{\prime}, \text { w }}$ is a finite $H$-module follows also from a general theorem on analytic algebras, [11, Theorem 1, p. 10]. 
theorem of the primitive element [14, p. 84] implies that $H\left(x_{0}, x_{1}\right)=H\left(x_{2}\right)$ for some $x_{2} \in \tilde{\mathcal{O}}_{\Omega^{\prime}, \mathrm{w}}$. But then $x_{2}$ has larger degree over $\tilde{H}$ than $x_{0}$, a contradiction. Consequently, $\tilde{\mathcal{O}}_{\Omega^{\prime}, \mathrm{w}}=\tilde{H}\left(x_{0}\right)$. This proves (ii) and completes the lemma.

I.3. Corollary. If $\Phi \in \mathscr{M}\left(\Delta, U^{N}\right)$ for some $\Delta \in \mathscr{K}_{N}$, if $\Omega$ is a neighborhood of $U^{N}$ such that $\Phi$ maps $\Omega^{\prime}=\Phi^{-1}(\Omega)$ properly onto $\Omega$, and if $\Phi$ has multiplicity $\lambda$, then the sheaf $\mathscr{S}$ is free of rank $\lambda$ on a neighborhood of $\bar{U}^{N}$.

Proof. This is simply the fact that a locally free sheaf on a neighborhood of a closed polydisc is actually free over some neighborhood of the polydisc. This follows from Cartan's lemma on holomorphic matrices and is to be found in $[10$, p. 86] where, however, it is formulated in terms of vector bundles. The relation between vector bundles and locally free sheaves is discussed in [5].

We can now prove the main result of this section.

I.4. THEOREM. If $\Delta \in \mathscr{K}_{N}$ and if $\Phi \in \mathscr{M}\left(\Delta, U^{N}\right)$ has multiplicity $\lambda$, then there exist functions $F_{1}, \ldots, F_{\lambda}$, holomorphic in a neighborhood of $\bar{\Delta}$, such that every $f$ holomorphic in $\Delta$ has a unique representation of the form

$$
f=\sum_{i=1}^{\lambda}\left(g_{i} \circ \Phi\right) F_{i}
$$

where $g_{1}, \ldots, g_{\lambda}$ are holomorphic in $U^{N}$.

Moreover, if $f \in H^{\infty}(\Delta)$ then each $g_{i} \in H^{\infty}\left(U^{N}\right)$. If $f \in A(\Delta)$, then each $g_{i} \in A\left(U^{N}\right)$.

Proof. By Corollary I.3, there is an open polydisc $\Omega \supset \bar{U}^{N}$ such that, setting $\Omega^{\prime}=\Phi^{-1}(\Omega)$, the sheaf $\mathscr{S}$ of Lemma I.2 is isomorphic to $\left(\mathcal{O}_{\Omega}\right)^{\lambda}$. Hence there are sections $\widetilde{F}_{1}, \ldots, \widetilde{F}_{\lambda} \in \Gamma(\Omega, \mathscr{S})$ with the following property: if $V$ is open, $V \subset \Omega$, then every $f \in \Gamma(V, \mathscr{S})$ is uniquely expressible as $\sum g_{i} \tilde{F}_{i}$, with $g_{i} \in \Gamma\left(V, \mathcal{O}_{V}\right)=\mathcal{O}(V)$.

If we apply this to $V=U^{N}$, lift the statement to $\Delta$ by means of $\Phi$, and let $F_{i}$ be the element of $\mathcal{O}\left(\Omega^{\prime}\right)$ which corresponds to the section $\widetilde{F}_{i} \in \Gamma(\Omega, \mathscr{S})$, we obtain (2).

If we apply the same statement to $V=\Omega$, we obtain an analogue (2') of (2), with $f \in \mathcal{O}\left(\Omega^{\prime}\right), g_{i} \in \mathcal{O}(\Omega)$. If $z \in \Omega$ is such that $\Phi^{-1}(\Phi(z))$ consists of $\lambda$ distinct points $w_{1}, \ldots, w_{\lambda}$, then $\left(2^{\prime}\right)$ gives

$$
f\left(w_{k}\right)=\sum_{i=1}^{\lambda} g_{i}(\Phi(z)) F_{i}\left(w_{k}\right) \quad(k=1, \ldots, \lambda) .
$$

Proper choice of $f \in \mathcal{O}\left(\Omega^{\prime}\right)$ shows that the ordered $\lambda$-tuple $\left(f\left(w_{1}\right), \ldots, f\left(w_{\lambda}\right)\right)$ can be any point of $C^{\lambda}$. The matrix $\left(F_{i}\left(w_{k}\right)\right)$ must therefore have rank $\lambda$ whenever $w_{1}, \ldots, w_{\lambda}$ are distinct.

This last condition holds for every $z$ in a certain neighborhood of $T^{N}$, since $\Phi$ is a local homeomorphism at every point of $\Phi^{-1}\left(T^{N}\right)$. Thus there is a neighborhood $Y$ of $\Phi^{-1}\left(T^{N}\right)$ in which $\operatorname{det}\left(F_{i}\left(w_{k}\right)\right)$ is bounded away from zero.

Now suppose $f \in H^{\infty}(\Delta)$. The equations ( $\left(3^{\prime}\right)$ have analogues (3), with $g_{i} \in \mathcal{O}\left(U^{N}\right)$. For $z \in \Delta \cap Y$, (3) can be solved for $g_{i}(\Phi(z))$, by Cramer's rule. The form of the 
solution shows that each $g_{i}$ is bounded in $\Phi(\Delta \cap Y)$. This latter set contains all $\left(\zeta_{1}, \ldots, \zeta_{N}\right)$ with $r<\left|\zeta_{j}\right|<1$ for some $r$ and all $j$. Hence $g_{\iota} \in H^{\infty}\left(U^{N}\right)$.

If, in addition, $f$ is continuous on $\Delta \cup \Phi^{-1}\left(T^{N}\right)$, then the above application of Cramer's rule shows that each $g_{i}$ extends to a function continuous on $\Phi(\Delta \cap Y) \cup$ $T^{N}$. It follows that $g_{i}$ is continuous on $U^{N} \cup T^{N}$. Hence $g_{i} \in A\left(U^{N}\right)$.

This completes the proof of the theorem. We note, incidentally, that the last paragraph of the proof shows that if $g$ is holomorphic in $\Delta$ and continuous on $\Delta \cup \Phi^{-1}\left(T^{N}\right)$, then $g \in A(\Delta)$.

Every $\Phi \in \mathscr{M}\left(\Delta, U^{N}\right)$ is, by definition, a local homeomorphism at each point of $\Phi^{-1}\left(T^{N}\right)$. We now show by an example that Theorem I.4 can fail (even for $N=1$ ) if $\Phi$ fails to be a local homeomorphism at just one point of $\Phi^{-1}\left(T^{N}\right)$.

I.5. Example. For $z \in C$, put $\Phi(z)=\left(z^{2}-1\right)^{-1}$, and regard $\Phi$ as a map of the Riemann sphere $S$ into itself. The curve $\gamma$ on which $|\Phi|=1$ is shaped like an infinity sign, and it meets the imaginary axis in only one point, namely the origin, which is a double point of the curve. Let $\Delta$ be the component of $S \mid \gamma$ which contains the point at infinity. Thus $\Delta=\Phi^{-1}(U)$, where $U$ is the open unit disc in the plane, and $\Phi$ maps $\Delta$ onto $U$ in a two-to-one fashion. The mapping $\Phi$ is a local homeomorphism at every point of the boundary $\gamma$ of $\Delta$, except zero. We shall show that $A(\Delta)$ is not a finitely generated module over $\Phi^{*} A(U)$.

Suppose, on the contrary, that $B_{1}, \ldots, B_{M} \in A(\Delta)$, and that every $F \in A(\Delta)$ has a (not necessarily unique) representation of the form

$$
F(z)=\sum_{i=1}^{M} B_{i}(z) f_{i}\left(\left(z^{2}-1\right)^{-1}\right) \quad\left(z \in \Delta, f_{i} \in A(U)\right) .
$$

Let $L: A(U)^{M} \rightarrow A(\Delta)$ be given by $L\left(f_{1}, \ldots, f_{M}\right)=\sum B_{j} f_{j} \circ \Phi$; our hypothesis is that $L$ is onto though not necessarily one-to-one.

If $G \in H^{\infty}(\Delta)$, there exists a bounded sequence $\left\{G_{n}\right\}$ in $A(\Delta)$ which converges uniformly on compacta in $\Delta$ to $G$. To see this, let $\psi: \Delta \rightarrow U$ be a conformal (one-to-one) mapping such that

$$
\lim _{t \rightarrow 0^{+}} \psi(i t)=1 \text { and } \lim _{t \rightarrow 0^{+}} \psi(i t)=-1
$$

Let $\phi: U \rightarrow \Delta$ be inverse to $\psi$. The mapping $\psi$ extends continuously to $\bar{\Delta} \backslash\{0\}$, and $\phi$ extends continuously to $\bar{U}$. Since $G \circ \phi \in H^{\infty}(U)$, there is a bounded sequence $\left\{g_{n}\right\}$ in $A(U)$ which converges uniformly on compacta in $U$ to $G \circ \phi$. Define $\tilde{g}_{n}$ by $\tilde{g}_{n}(z)=g_{n}(z)\left(1-z^{2}\right)^{1 / n}$. The sequence $\left\{\tilde{g}_{n}\right\}$ is again a bounded sequence in $A(U)$ which converges to $G \circ \phi$ uniformly on compacta in $U$. Since the functions $\tilde{g}_{n}$ vanish at 1 and $-1, G_{n}=\tilde{g}_{n} \circ \psi$ is a well-defined element of $A(\Delta)$. Then $\left\{G_{n}\right\}$ is a bounded sequence in $A(\Delta)$ which converges uniformly on compacta in $\Delta$ to $G$.

Since the operator $L$ is onto, the open mapping theorem, together with a simple normal families argument, shows that if $F \in H^{\infty}(\Delta)$, then $F$ can be expressed in the form (4) with suitable $f_{1}, \ldots, f_{M} \in H^{\infty}(U)$. We will obtain our contradiction by applying this fact to a particular $f \in H^{\infty}(\Delta)$. 
The function $\Phi$ is negative on the imaginary axis, and $\lim _{t \rightarrow 0} \Phi(i t)=-1$. Let $\left\{\tau_{n}\right\}_{n=1}^{\infty}$ be a sequence in $(-1,0)$ which decreases to -1 . For each $n$, let $\Phi^{-1}\left(\tau_{n}\right)=$ $\left\{\sigma_{n}^{+}, \sigma_{n}^{-}\right\}, \sigma_{n}^{+}$above the real axis, $\sigma_{n}^{-}$below. If $\tau_{n}$ approaches -1 fast enough, there will exist an $F_{0} \in H^{\infty}(\Delta)$ such that $F_{0}\left(\sigma_{n}^{+}\right)=+1, F_{0}\left(\sigma_{n}^{-}\right)=-1$. (One way to obtain such an $F_{0}$ is to note that if $\tau_{n}$ tends to -1 fast enough, the set $\left\{\sigma_{n}^{+}\right\}_{n=1}^{\infty} \cup\left\{\sigma_{n}^{-}\right\}_{n=1}^{\infty}$ will be an interpolation set for $H^{\infty}(\Delta)$. In this connection, see [7].) By the last paragraph, the function $F_{0}$ can be written in the form (4), and we have, for all $n$,

$$
1=F_{0}\left(\sigma_{n}^{+}\right)=B_{1}\left(\sigma_{n}^{+}\right) f_{1}\left(\tau_{n}\right)+\cdots+B_{M}\left(\sigma_{n}^{+}\right) f_{M}\left(\tau_{n}\right)
$$

and

$$
-1=F_{0}\left(\sigma_{n}^{-}\right)=B_{1}\left(\sigma_{n}^{-}\right) f_{1}\left(\tau_{n}\right)+\cdots+B_{M}\left(\sigma_{n}^{-}\right) f_{M}\left(\tau_{n}\right) .
$$

Since the functions $f_{j}$ are bounded in $U$, there exists a subsequence of $\left\{\tau_{n}\right\}$, call it $\left\{\tau_{n}^{\prime}\right\}$ with the property that for each $j$,

$$
\lim _{n} f_{j}\left(\tau_{n}^{\prime}\right)=\alpha_{j}
$$

exists. The functions $B_{j}$ are continuous at 0 , so if we take limits along the sequence $\left\{\tau_{n}^{\prime}\right\}$ in (5) and (6), we are led to the contradiction that $-1=\sum B_{j}(0) \alpha_{j}=1$. Thus $A(\Delta)$ is not finitely generated over $\Phi^{*} A(U)$.

Let us observe that in this example $H^{\infty}(\Delta)$ is a free module of rank two over $\Phi^{*} H^{\infty}(U)$. Denote by $q$ one of the branches of $\left(z^{2}-1\right)^{-1 / 2}$ holomorphic in $\Delta$. The function $q$ effects a conformal (one-to-one) mapping of $\Delta$ onto $U$. Each $F \in H^{\infty}(\Delta)$ is uniquely expressible in the form $F=f \circ q, f \in H^{\infty}(U)$. If $f(\zeta)=$ $\sum_{k=0}^{\infty} b_{k} \zeta^{k}$, then the functions $f_{1}$ and $f_{2}$ defined by

$$
f_{1}(\zeta)=\sum_{k=0}^{\infty} b_{2 k+1} \zeta^{k} \text { and } f_{2}(\zeta)=\sum_{k=0}^{\infty} b_{2 k} \zeta^{k}
$$

are both in $H^{\infty}(U)$, and we have $f(\zeta)=\zeta f_{1}\left(\zeta^{2}\right)+f_{2}\left(\zeta^{2}\right)$. Consequently, $F=q f_{1} \circ \Phi+$ $f_{2} \circ \Phi$. Since the $f_{1}$ and $f_{2}$ in this decomposition are uniquely determined by $F$, it follows that $\{1, q\}$ is a free basis for $H^{\infty}(\Delta)$ over $\Phi^{*} H^{\infty}(U)$. Of course, $q$ is not continuous on $\bar{\Delta}$. Our previous argument shows that $H^{\infty}(\Delta)$ cannot be generated as a module over $\Phi^{*} H^{\infty}(U)$ by finitely many elements of $A(\Delta)$.

We now turn to some corollaries of Theorem I.4.

I.6. Corollary. (a) If $f \in A(\Delta), \Delta \in \mathscr{K}_{N}$, then $f$ can be approximated uniformly on $\bar{\Delta}$ by functions holomorphic on a neighborhood of $\bar{\Delta}$.

(b) If $F \in H^{\infty}(\Delta), \Delta \in \mathscr{K}_{N}$, there is a bounded sequence in $A(\Delta)$ which converges uniformly on compacta in $\Delta$ to $F$.

Proof. Let $\Phi \in \mathscr{M}\left(\Delta, U^{N}\right)$ and let $G_{1}, \ldots, G_{\lambda} \in \mathcal{O}(\bar{\Delta})$ constitute a free basis for $H^{\infty}(\Delta)$ over $\Phi^{*} H^{\infty}\left(U^{N}\right)$. If $F \in H^{\infty}(\Delta)$, write

$$
F=\sum G_{j} \cdot\left(f_{j} \circ \Phi\right), \quad f_{j} \in H^{\infty}\left(U^{N}\right) .
$$


For $m=2,3, \ldots$, let $f_{j}^{(m)}(z)=f,((1-1 / m) z)$ for $z \in U^{N}$, and set

$$
F_{m}=\sum G_{j} \cdot\left(f_{j}^{(m)} \circ \Phi\right) \text {. }
$$

The sequence $\left\{F_{m}\right\}$ is a bounded sequence of functions holomorphic on $\bar{\Delta}$, and it converges uniformly on compacta in $\Delta$ to $F$. If $F \in A(\Delta)$, then the functions $f_{j}$ lie in $A\left(U^{N}\right)$, and the sequence $\left\{F_{m}\right\}$ converges uniformly on $\bar{\Delta}$ to $F$.

It would be of interest to determine whether or not given $F \in H^{\infty}(\Delta)$, the sequence $\left\{F_{m}\right\}$ can be chosen to satisfy $\left\|F_{m}\right\| \leqq\|F\|$. This may be the case, but we have not proved it.

As noted in [13], theorems like Theorem I.4 can be used to prove certain extension theorems for functions in $A(\Delta)$ and $H^{\infty}(\Delta)$ when $\Delta$ is embedded in a polydisc. We have the following fact.

I.7. THEOREM. Let $\Delta \in \mathscr{K}_{N}$, let $\Omega$ be a neighborhood of $\bar{\Delta}$ which is carried biholomorphically onto an analytic submanifold $V$ of a neighborhood $\Omega^{\prime}$ of $\bar{U}^{M}$ by the map $\Psi$, and let $\pi: U^{M} \rightarrow U^{N}$ be the projection which takes $\left(z_{1}, \ldots, z_{M}\right)$ to $\left(z_{1}, \ldots, z_{N}\right)$. If $\Delta=\Psi^{-1}\left(V \cap U^{M}\right)$, and if $\pi \circ \Psi \in \mathscr{M}\left(\Delta, U^{M}\right)$, then there exists a bounded linear operator $E: H^{\infty}(\Delta) \rightarrow H^{\infty}\left(U^{M}\right)$ which carries $A(\Delta)$ into $A\left(U^{M}\right)$ and which is an extension operator in the sense that $(E f) \circ \Phi=f$ for all $f \in H^{\infty}(\Delta)$.

Proof. Let $F_{1}, \ldots, F_{\lambda} \in \mathcal{O}(\bar{\Delta})$ be a free basis for $H^{\infty}(\Delta)$ over $(\pi \circ \Psi)^{*} H^{\infty}\left(U^{N}\right)$. Since the functions $F_{j}$ are holomorphic on a neighborhood of $\bar{\Delta}$, and since $V$ is an analytic submanifold of $\Omega^{\prime}$, it follows [5, p. 245] that for some functions $G_{1}, \ldots, G_{\lambda}$ holomorphic on a neighborhood of $\bar{U}^{M}$, we have $F_{j}=G_{j} \circ \Psi$. We construct $E$ as follows: If $f \in H^{\infty}(\Delta)$ and $f=\sum F_{j}\left(f_{j} \circ \pi \circ \Psi\right)$ with $f_{j} \in H^{\infty}\left(U^{N}\right)$, define $E f \in H^{\infty}\left(U^{M}\right)$ by $E f=\sum G_{j} f_{j} \circ \pi$. The operator $E$ so defined is plainly linear and continuous. The choice of the functions $G_{j}$ and $f_{j}$ shows that $E f \circ \Psi=f$.

If we set $\Delta^{\prime}=\Psi(\Delta)$, this theorem shows that each bounded holomorphic function $f$ on $\Delta^{\prime}$ extends to a bounded holomorphic function $F$ on $U^{M}$ and, moreover, that if $f$ has continuous boundary values, the extension $F$ will also have continuous boundary values. As noted in [13, Example III.6] the norm of the operator will, in general, exceed one. (Unfortunately, this example is not correct as stated, for the functions $\psi_{\delta}$ are not one-to-one. We obtain a correct example if we redefine $\psi_{\delta}$ by means of

$$
\psi_{\delta}(\zeta)=\left(\zeta^{2}, \zeta^{3},\left(\frac{\zeta-\delta}{1-\delta \zeta}\right)^{3}\right)
$$

Theorem II.9 of the present paper shows that no example with just two Blaschke products can exist.)

In general, $E(1)$ is not the function identically one on $U^{N}$, for the functions $G_{j}$ can very well have common zeros in $U^{N}$.

In connection with this theorem, we should point out that in [3] Bishop has shown the existence of linear solutions to certain extension problems. See especially Theorem $7 . \mathrm{II}_{1}$ and the note added in proof. 
I.8. Corollary. If $\Delta^{\prime}=\Psi(\Delta)$, the ideal $I=\left\{f \in H^{\infty}\left(U^{M}\right): f \mid \Delta^{\prime}=0\right\}$ is a direct summand in $H^{\infty}\left(U^{M}\right)$, and $I \cap A\left(U^{M}\right)$ is a direct summand in $A\left(U^{M}\right)$.

Proof. Given $f \in H^{\infty}\left(U^{M}\right)$, define $P f$ by

$$
P f=f-E(f \circ \Psi) \text {. }
$$

The operator $P$ is a continuous projection in $H^{\infty}\left(U^{M}\right)$ whose range is the ideal $I$ and which carries $A\left(U^{M}\right)$ onto $I \cap A\left(U^{M}\right)$. The existence of such projections implies the corollary.

II. Embedding polydises in polydiscs. In this section we will investigate the properties of mappings which embed polydiscs in higher dimensional polydiscs. We begin with a pair of lemmas.

II.1. Lemma. Suppose $\Omega$ is a connected open set in $C^{N},\left\{g_{j}\right\}$ is a sequence in $\mathcal{O}(\Omega)$ each member of which is bounded by one in modulus, and $\lim _{j \rightarrow \infty} g_{j}\left(z_{0}\right)=\alpha$ for some $z_{0} \in \Omega$ and some $\alpha$ with $|\alpha|=1$. Then $\lim g_{j}(z)=\alpha$ uniformly on compacta in $\Omega$.

A standard normal families argument guarantees the conclusion for some subsequence of $\left\{g_{j}\right\}$; the lemma shows that it is unnecessary to pass to a subsequence.

Proof. If $K$ is compact in $\Omega$, there exists a compact set $H \subset U$, the unit disc in $C$, such that if $g \in \mathcal{O}(\Omega)$ vanishes at $z_{0}$ and is bounded by one on $\Omega$, then $g(K) \subset H$. Set

$$
\phi_{w}(z)=(z-w) /(1-\bar{w} z), \quad \psi_{w}(z)=(z+w) /(1+\bar{w} z)
$$

and $f_{j}(z)=\phi_{w_{j}}\left(g_{j}(z)\right)$ where $w_{j}=g_{j}\left(z_{0}\right)$. Then $f_{j}(K) \subset H$, and since $g_{j}=\psi_{w j} \circ f_{j}$, we see that $g_{j}(K) \subset \psi_{w_{j}}(H)$. Since $w_{j} \rightarrow \alpha,|\alpha|=1$, the sequence $\left\{\psi_{w_{j}}\right\}$ converges to $\alpha$ uniformly on compacta in $U$, and the result follows.

II.2. LeMMA. If $\Phi: U^{N} \rightarrow U^{M}$ is a proper holomorphic map, then $N \leqq M$.

Proof. If $M<N$, then since $\Phi$ is a closed mapping, a result from dimension theory [8, p. 91] provides a point $z \in U^{M}$ such that $\Phi^{-1}(z)$ is of positive dimension. Since $\Phi$ is proper and holomorphic, $\Phi^{-1}(z)$ is a compact subvariety of $U^{N}$. Since compact subvarieties of $U^{N}$ are necessarily finite sets, we have a contradiction, and the lemma is proved.

The proper holomorphic maps of $U$ into $U$ are the finite Blaschke products. Our next theorem shows that proper holomorphic maps of a $U^{k}$ into a $U^{n}$ are also of a rather special form. If $f \in \mathcal{O}\left(U^{k}\right)$ and $z \in T^{k}$, we shall denote by $f^{*}(z)$ the limit $\lim _{r \rightarrow 1}-f(r z)$ provided this limit exists. If $f$ is bounded, $f^{*}(z)$ exists for almost all $z \in T^{k}$. (See, e.g., [15].)

II.3. TheOREM. Let $\Phi=\left(\phi, \ldots, \phi_{n}\right)$ be a proper holomorphic map of $U^{k}$ into $U^{n}$. Then $k \leqq n$, and the functions $\phi_{1}, \ldots, \phi_{n}$ can be so permuted that for $1 \leqq j \leqq k$,

(1) $\phi_{j}$ depends only on $z_{j}$ and is nonconstant, and

(2) $\left|\phi_{j}^{*}\right|=1$ on a set of positive measure in T, the unit circle. Moreover, if one of the following conditions (a), (b) or (c) is satisfied, then the functions $\phi_{1}, \ldots, \phi_{k}$ are finite Blaschke products: 
(a) $k=n$.

(b) $\Phi$ is continuous on $\bar{U}^{k}$ and $\Phi\left(T^{k}\right) \subset T^{n}$.

(c) $\Phi$ is holomorphic on a neighborhood of $\bar{U}^{k}$.

Simple examples show that a proper holomorphic map $\Phi: U^{k} \rightarrow U^{n}$ need not satisfy any of the conditions (a), (b) or (c). For instance, let $\phi_{1}$ be a conformal, one-to-one map of $U$ onto the right half of $U$ so that $\phi_{1}(1)=1, \phi_{1}(i)=i, \phi_{1}(-i)=-i$ and let $\phi_{2}$ be a conformal map of $U$ onto the left half of $U$ so that $\phi_{2}(-1)=-1$, $\phi_{2}(-i)=-i$, and $\phi_{2}(i)=i$. Then $\Phi=\left(\phi_{1}, \phi_{2}\right)$ is a proper map of $U$ to $U^{2}$ but neither $\phi_{1}$ nor $\phi_{2}$ is a finite Blaschke product.

Proof of the Theorem. We know from Lemma II.2 that $k \leqq n$.

If $z=\left(z_{1}, \ldots, z_{k}\right)$, set $z^{\prime}=\left(z_{2}, \ldots, z_{k}\right)$, and put $0^{\prime}=(0, \ldots, 0) \in C^{k-1}$. For each $i$ and almost all $\zeta \in T$, the limit

$$
c_{i}(\zeta)=\lim _{r \rightarrow 1-} \phi_{i}\left(r \zeta, 0^{\prime}\right)
$$

exists, and since $\Phi$ is proper, $\left|c_{i}(\zeta)\right|=1$ for at least one $i$. Let $E_{i}=\left\{\zeta \in T:\left|c_{i}(\zeta)\right|=1\right\}$. Then $E_{i}$ has positive measure for at least one $i$ since $E_{1} \cup \cdots \cup E_{k}$ covers almost all of $T$. By re-indexing if necessary, we may suppose $E_{1}$ to have positive measure, so that

$$
\lim _{r \rightarrow 1^{-}} \phi_{1}\left(r \zeta, 0^{\prime}\right)=c_{1}(\zeta)
$$

is of modulus one on a set of $\zeta$ 's of positive measure. By Lemma II.1, applied to $U^{k-1}$, it follows that

$$
\lim _{r \rightarrow 1^{-}} \phi_{1}\left(r \zeta, z^{\prime}\right)=c_{1}(\zeta)
$$

for all $z^{\prime} \in U^{k-1}, \zeta \in E_{1}$. If we define $g_{z^{\prime}}(\lambda)=\phi_{1}\left(\lambda, z^{\prime}\right)$, then $g_{z^{\prime}} \in H^{\infty}(U)$ for each $z^{\prime} \in U^{k-1}$, and (3) implies that the radial limit of $g_{z^{\prime}}-g_{w^{\prime}}$ vanishes on $E_{1}$ whenever $z^{\prime}, w^{\prime} \in U^{k-1}$. Since $E_{1}$ has positive measure, we have $g_{z^{\prime}}=g_{w^{\prime}}$, i.e., $\phi_{1}\left(\lambda, z^{\prime}\right)$ depends only on $\lambda$. If $\phi_{1}$ were constant, it would have to be of modulus one which is impossible since $\Phi$ carries $U^{k}$ into $U^{n}$. The variables $z_{2}, \ldots, z_{n}$ can be dealt with in a similar way, so we have (1) and (2) of the theorem.

If $k=n$, it follows, after a permutation of indices, that

$$
\Phi\left(z_{1}, \ldots, z_{k}\right)=\left(\phi_{1}\left(z_{1}\right), \ldots, \phi_{k}\left(z_{k}\right)\right)
$$

and in particular that $\Phi\left(z_{1}, 0, \ldots, 0\right)=\left(\Phi_{1}\left(z_{1}\right), \phi_{2}(0), \ldots, \phi_{k}(0)\right)$. Since $\Phi$ is proper, $\phi_{1}$ must be a proper holomorphic map of $U$ to $U$, i.e., it must be a finite Blaschke product.

We now consider the case that $\Phi$ is holomorphic on a neighborhood of $\bar{U}^{k}$. The fact that in this case $\phi_{1}, \ldots, \phi_{k}$ must be finite Blaschke products is an immediate consequence of the following result.

II.4. LEMMA. If $f$ is holomorphic on a neighborhood of $\bar{U}^{k}$ and if $|f|=1$ on a set of positive measure in $T^{k}$, then $|f|$ is identically one on $T^{k}$. 
Proof. Define a map $Q: R^{k} \rightarrow T^{k}$ by

$$
Q\left(t_{1}, \ldots, t_{k}\right)=\left(e^{i t_{1}}, \ldots, e^{i t_{k}}\right) .
$$

The function $1-|f \circ Q|^{2}=1-(f \circ Q)(\overline{f \circ Q})$ is real analytic on $R^{k}$ and vanishes on a set of positive measure. Consequently, it vanishes identically, and thus $|f|=1$ on $T^{k}$.

It remains only to consider the case (b). Under the hypotheses of (b), each $\phi_{j}$ is continuous on $\bar{U}^{k}$ and has modulus one on $T^{k}$. For $1 \leqq j \leqq k, \phi_{j}$ depends only on $z_{j}$ and it follows that $\phi_{j}$ must, in fact, be a finite Blaschke product. This concludes the proof of the theorem.

It is interesting to observe that if $\Phi$ satisfies condition (b), it automatically satisfies condition (c) as our next lemma shows.

II.5. LEMMA. If $f \in A\left(U^{k}\right)$ has modulus one on $T^{k}$, then $f$ is holomorphic on a neighborhood of $\bar{U}^{k}$.

Proof. By Theorems 2.1 and 2.2 of [12], $f=P / Q, P$ and $Q$ relatively prime polynomials, $Q$ free of zeros in $U^{k}$. Equation 2.1.2 of the same reference implies that $Q$ is free of zeros on $T^{k}$. It follows that $Q$ is free of zeros in $\bar{U}^{k}$, for otherwise $1 / Q$ would violate the maximum modulus theorem.

Using Theorem II.3 and results from §I, we can establish the following fact.

II.6. THEOREM. Let $\Phi=\left(\phi_{1}, \ldots, \phi_{N}\right)$ be a holomorphic map of a neighborhood of $\bar{U}^{k}$ into $C^{N}$ which is proper on $U^{k}$, nonsingular and one-to-one on $\bar{U}^{k}$ and which carries $U^{k}$ into $U^{N}$. Then there is a continuous linear operator $E: H^{\infty}\left(U^{k}\right) \rightarrow$ $H^{\infty}\left(U^{N}\right)$ which carries $A\left(U^{k}\right)$ into $A\left(U^{N}\right)$ and which satisfies $E f \circ \Phi=f$.

Proof. By Theorem II.3, we can reindex the functions $\phi_{j}$ so that

$$
\phi_{j}(z)=B_{j}\left(z_{j}\right) \quad i \leqq j \leqq k,
$$

where each $B_{j}$ is a nonconstant finite Blaschke product.

Let $\Psi: U^{k} \rightarrow U^{k}$ be given by $\Psi(z)=\left(B_{1}\left(z_{1}\right), \ldots, B_{k}\left(z_{k}\right)\right)$. We assert that the $\Psi$ so defined is in $\mathscr{M}\left(U^{k}, U^{k}\right)$. It surely is holomorphic on a neighborhood of $\bar{U}^{k}$. It is nonsingular at each point of $T^{k}$, for the Jacobian det $\left(\partial \phi_{j} / \partial z_{k}\right)$ is simply $B_{1}^{\prime}\left(z_{1}\right) \cdots B_{k}^{\prime}\left(z_{k}\right)$, and since each of the derivatives $B_{j}^{\prime}$ is zero free on the unit circle, this Jacobian cannot vanish on $T^{k}$. If we let $D_{R}=\left\{\left(z_{1}, \ldots, z_{k}\right):\left|B_{j}\left(z_{j}\right)\right|<R\right\}$, then for $R$ larger than but sufficiently near one, $\Psi$ will be holomorphic on $D_{R}$ and will map $D_{R}$ properly onto a neighborhood of $\bar{U}^{k}$. Thus $\Psi \in \mathscr{M}\left(U^{k}, U^{k}\right)$.

Since $\Psi \in \mathscr{M}\left(U^{k}, U^{k}\right)$, Theorem I.4 implies that $A\left(U^{k}\right)$ is a free module of rank $\lambda$, the multiplicity of $\Psi$, over $\Psi^{*} A\left(U^{k}\right)$. Let $\left\{F_{1}, \ldots, F_{\lambda}\right\}$ be a free basis for $A\left(U^{k}\right)$ over $\Psi^{*} A\left(U^{k}\right)$, each $F_{j}$ holomorphic on a neighborhood of $\bar{U}^{k}$. Then $\left\{F_{1}, \ldots, F_{\lambda}\right\}$ is also a free basis for $H^{\infty}\left(U^{k}\right)$ over $\Psi^{*} H^{\infty}\left(U^{k}\right)$.

If we make $R$ small enough, the neighborhood $D_{R}$ of the next-to-last paragraph will be contained in the domain of definition of each of the functions $F_{j}$ and also in 
that of all the $\phi_{j}$. Since $\Psi$ carries $D_{R}$ properly onto a neighborhood $\Omega$ of $\bar{U}^{k}$, it follows that $\Phi$ will carry $D_{R}$ properly into $\Omega \times C^{N-k}$.

Since $\Phi$ is nonsingular at each point of $\bar{U}^{k}$, it is nonsingular on $D_{R}$ if $R$ is small enough. Finally, if $R$ is small enough, $\Phi$ will be one-to-one on $D_{R}$. If not there is a sequence $\left\{R_{n}\right\}$ which decreases to one, and for each $n$ a pair of points $z_{n}$ and $z_{n}^{\prime}$ in $D_{R_{n}}, z_{n} \neq z_{n}^{\prime}$, such that $\Phi\left(z_{n}^{\prime}\right)=\Phi\left(z_{n}\right)$. By passing to subsequences, we may suppose $\left\{z_{n}\right\}$ and $\left\{z_{n}^{\prime}\right\}$ to converge to $z_{0}$ and $z_{0}^{\prime}$ respectively. We shall have $z_{0}, z_{0}^{\prime} \in \bar{U}^{k}$ and $\Phi\left(z_{0}\right)=\Phi\left(z_{0}^{\prime}\right)$. Since $\Phi$ is one-to-one on $\bar{U}^{k}$, we must have $z_{0}=z_{0}^{\prime}$. However, since $\Phi$ is nonsingular at each point of $\bar{U}^{k}$, there is a neighborhood $W$ of $z_{0}$ in $C^{k}$ on which $\Phi$ is one-to-one. Since $z_{n}$ and $z_{n}^{\prime}$ are eventually in $W$, we have a contradiction. Thus $\Phi$ must be one-to-one on $D_{R}$ for $R$ near one. Consequently if $R$ is near enough to one, the set $\Phi\left(D_{R}\right)$ in $\Omega \times C^{N-k}$ will in fact be a submanifold, say $M$, and $\Phi: D_{R} \rightarrow M$ will be a biholomorphic map.

Thus for some choice of $G_{1}, \ldots, G_{\lambda} \in \mathcal{O}\left(\Omega \times C^{N-k}\right)$ we have $F_{j}=G_{j} \circ \Phi$. The operator $E$ defined by $E f=\sum G_{j} \tilde{f}_{j}$, if $f=\sum F_{j} f_{j} \circ \Psi$ and $\tilde{f}_{j}\left(z_{1}, \ldots, z_{n}\right)=f_{j}\left(z_{1}, \ldots, z_{k}\right)$ has the desired properties.

Thus, if we embed $U^{k}$ in $U^{N}$ as a submanifold and if the embedding satisfies certain regularity conditions at the boundary, bounded holomorphic functions on the embedded $U^{k}$ extend to bounded holomorphic functions on $U^{N}$. It is natural to ask if the boundary regularity is necessary. The following example shows that some condition is necessary.

II.7. EXAMPLE. We will construct a proper nonsingular one-to-one map $\Phi$ from $U$ to $U^{2}$ such that for some $f \in H^{\infty}\left(U^{2}\right)$ there is no $F \in H^{\infty}(U)$ with $f=F \circ \Phi$.

Let $S$ be the spiral

$$
\left\{r e^{i \theta}: r=1-1 / \theta, \pi \leqq \theta<\infty\right\} .
$$

Let $\Omega=U \mid S$, and let $h$ be a conformal (1-1) mapping of $U$ onto $\Omega$. The map $h$ can be chosen so that it is continuous on $\bar{U} \mid\{1\}$. Let $0<r_{1}<r_{2}<\cdots$ be the points at which $S$ meets $(0,1)$. Fix $n$ for the moment, and put $\alpha(\varepsilon)=h^{-1}\left(r_{n}+\varepsilon\right), \beta(\varepsilon)=$ $h^{-1}\left(r_{n}-\varepsilon\right)$ where $\varepsilon$ is small and positive. As $\varepsilon \rightarrow 0, \alpha(\varepsilon)$ and $\beta(\varepsilon)$ tend to distinct points of $\partial U$. Hence we can choose $\varepsilon_{n}>0$ so small that the following properties hold: If $\xi_{n}=r_{n}+\varepsilon_{n}, \eta_{n}=r_{n}-\varepsilon_{n}, h\left(\alpha_{n}\right)=\xi_{n}$ and $h\left(\beta_{n}\right)=\eta_{n}$, then $1-\left|\alpha_{n}\right|<n^{-2}$, $1-\left|\beta_{n}\right|<n^{-2}$, and

$$
\left|g\left(\xi_{n}\right)-g\left(\eta_{n}\right)\right| \leqq\left(\left|\alpha_{n}-\beta_{n}\right| / n\right)\|g\|_{U}
$$

for all $g \in H^{\infty}(U)$. The choice of $h$ shows that $\alpha_{n}, \beta_{n} \rightarrow 1$ as $n \rightarrow \infty$.

Let $B$ be the Blaschke product whose zero set is $\left\{\alpha_{n}\right\}_{n=1}^{\infty} \cup\left\{\beta_{n}\right\}_{n=1}^{\infty}$, and define

$$
\Phi(z)=(B(z), h(z)) .
$$

Since $h$ is one-to-one, $h^{\prime}$ is zero free, so $\Phi$ is one-to-one and nonsingular. It is also proper, for as $z \rightarrow 1,|h(z)| \rightarrow 1$ and as $z \rightarrow e^{i \theta} \neq 1,|B(z)| \rightarrow 1$.

Suppose there exists $F \in H^{\infty}\left(U^{2}\right)$ such that $F(\Phi(z))=z$. Let $g(w)=F(0, w)$, $w \in U$. Since $\Phi(U)$ contains the points $\left(0, h\left(\alpha_{n}\right)\right)=\left(0, \xi_{n}\right)$ and $\left(0, h\left(\beta_{n}\right)\right)=\left(0, \eta_{n}\right)$ at 
which $F$ is $\alpha_{n}$ and $\beta_{n}$ respectively, the inequality (7) implies that $\|g\|_{U} \geqq n$ for all $n$, an impossibility since $F$ is assumed bounded.

In connection with this example, it should be mentioned that the disc $\Phi(U)$ is not the zero set of any $F \in H^{\infty}\left(U^{2}\right)$. The choice of the spiral $S$ shows that $r_{n}=$ $1-(2 n \pi)^{-1}$, so $\sum\left(1-r_{n}\right)=\infty$ whence $\sum\left(1-\left|\xi_{n}\right|\right)=\infty$. If $f \in H^{\infty}\left(U^{2}\right)$ vanishes on $\Phi(U)$, then $F\left(0, \xi_{n}\right)=0$ for all $n$. Hence, setting $g(\lambda)=F(0, \lambda), g\left(\xi_{n}\right)=0$ for all $n$. Since $g \in H^{\infty}(U)$ and since $\sum\left(1-\left|\xi_{n}\right|\right)=\infty$, it follows that $g$ vanishes identically, and so $F$ vanishes on the set $\{0\} \times U$. Thus $\Phi(U)$ is the zero set of no $F \in H^{\infty}\left(U^{2}\right)$.

II.8. REMARK. There are serious restrictions on the way in which a $U^{k}$ can be embedded in a $U^{N}$ if the embedding is required to be holomorphic on a neighborhood of $\bar{U}^{k}$. For instance, suppose $\Phi$ is holomorphic on $\bar{U}^{k}$ and carries $U^{k}$ properly into $U^{N}$. Then Corollary II.3 shows that $\Phi$ is of the form

$$
\Phi(z)=\left(B_{1}\left(z_{1}\right), \ldots, B_{k}\left(z_{k}\right), \phi_{k+1}(z), \ldots, \phi_{N}(z)\right)
$$

where the $B_{j}$ are finite Blaschke products. Consequently, if none of the $B_{j}$ are one-to-one, and if $N \leqq 2 k-1, \Phi$ cannot be nonsingular at every point of $U^{k}$.

Another result of this same nature is contained in the following theorem.

II.9. ThEOREM. Suppose $B_{1}$ and $B_{2}$ are finite Blaschke products of multiplicities $1+k_{1}$ and $1+k_{2}, k_{1}>0, k_{2}>0$. Then either $B_{1}^{\prime}$ and $B_{2}^{\prime}$ have a common zero in $U$ or else the pair $\left(B_{1}, B_{2}\right)$ does not separate points on $\bar{U}$.

Proof. Let $\alpha_{1}(z), \ldots, \alpha_{k_{1}}(z)$ be the points other than $z$ for which $B_{1}\left(\alpha_{t}(z)\right)=B_{1}(z)$. (If $B_{1}^{\prime}(z)=0$, we allow some of the $\alpha_{i}(z)$ to be $z$, the number to depend on the multiplicity of $B_{1}$ at $z$.) Define $\beta_{j}(z)$ in a similar way: $B_{2}\left(\beta_{j}(z)\right)=B_{2}(z)$. The function $R$ defined by

$$
R(z)=\prod_{i=1}^{k_{1}} \prod_{j=1}^{k_{2}}\left\{\alpha_{i}(z)-\beta_{j}(z)\right\}
$$

is holomorphic in a neighborhood of $\bar{U}$. If $|z|=1$, then $\left|\alpha_{i}(z)\right|=\left|\beta_{j}(z)\right|=1$, so

$$
\begin{aligned}
\bar{R} & =\prod_{i, j}\left(\bar{\alpha}_{i}-\bar{\beta}_{j}\right)=\prod_{i, j}\left(\frac{1}{\alpha_{i}}-\frac{1}{\beta_{j}}\right) \\
& =\prod_{i, j}\left(\beta_{j}-\alpha_{i}\right)\left(\prod_{i} \alpha_{i}\right)^{-k_{2}}\left(\prod_{j} \beta_{j}\right)^{-k_{1}} \\
& =(-1)^{k_{1} k_{2}} R\left(\prod_{i} \alpha_{i}\right)^{-k_{2}}\left(\prod_{j} \beta_{j}\right)^{-k_{1}} .
\end{aligned}
$$

Assume for the moment that $R$ has no zero on $|z|=1$. Then

$$
R(z)(\overline{R(z)})^{-1}=(-1)^{k_{1} k_{2}}\left(\prod_{i=1}^{k_{1}} \alpha_{i}(z)\right)^{k_{2}}\left(\prod_{j=2}^{k_{2}} \beta_{j}(z)\right)^{k_{1}} \quad(|z|=1) .
$$

As $z$ traverses the unit circle once in the positive direction, the argument of the right side increases by $2 \pi 2 k_{1} k_{2}$, and the argument of the left side increases by 
$2 \Delta_{|z|=1} \arg R$. Consequently $\Delta_{|z|=1} \arg R=2 \pi k_{1} k_{2}$ so that $R$ has $k_{1} k_{2}$ zeros in $U$. In any event, $R$ has a zero in $\bar{U}$, say $R\left(z_{0}\right)=0$. Hence there are $i$ and $j$ such that $\alpha_{i}\left(z_{0}\right)=\beta_{j}\left(z_{0}\right)$; let this common value be $w_{0}$. If $w_{0}=z_{0}$, then $B_{1}^{\prime}\left(z_{0}\right)=B_{2}^{\prime}\left(z_{0}\right)=0$. Otherwise we have distinct points $z_{0}, w_{0}$ which are not separated by $\left(B_{1}, B_{2}\right)$.

This theorem can be rephrased by saying that if two finite Blaschke products generate the Banach algebra $A(U)$ then one of them does by itself.

However, there exist three finite Blaschke products which generate $A(\bar{U})$ although no two of them do. See [13, Theorem IV.1].

III. Extending functions from embedded Reimann surfaces. In this short concluding section, we generalize Theorem II.1 of [13]. By a finite open Riemann surface we mean a Riemann surface $R$ obtained by deleting from a compact surface $R_{0}$ a finite collection of disjoint closed discs with analytic bounding curves.

III.1. TheOREM. Let $R$ be a finite open Riemann surface with boundary $\Gamma=$ $\Gamma_{1} \cup \cdots \cup \Gamma_{M}$, each $\Gamma_{j}$ an analytic simple closed curve. Let $\Phi: R \rightarrow U^{N}$ be a map which is holomorphic on some neighborhood $V$ of $\bar{R}$, which is proper on $R$, and which embeds $V$ as an analytic submanifold of a neighborhood of $\bar{U}^{N}$. Given $f \in A(R)$ $\left(H^{\infty}(R)\right)$, there is $F \in A\left(U^{N}\right)\left(H^{\infty}\left(U^{N}\right)\right)$ such that $F \circ \Phi=f$.

Let $\Phi=\left(\phi_{1}, \ldots, \phi_{N}\right)$. In [13], this theorem was proved under the additional assumption that one of the $\phi_{j}$ satisfies $\left|\phi_{j}\right| \equiv 1$ on $\Gamma$. Since such a $\phi_{j}$ lies in $\mathscr{M}(R, U)$, this case is also included in the results of our $\S I$.

The proof of the theorem depends on a simple lemma.

III.2. Lemma. If $\Phi=\left(\phi_{1}, \ldots, \phi_{N}\right)$ is as in the statement of the theorem, then for each $j$ there is a $k$ such that $\left|\phi_{k}\right| \equiv 1$ on $\Gamma_{j}$.

Proof. The mapping $\Phi$ is proper, so if $\zeta \in \Gamma_{j}$, there is $k(\zeta)=k$ such that $\left|\phi_{k}(\zeta)\right|=1$. Set $E_{k}=\left\{\zeta \in \Gamma_{j}:\left|\phi_{k}(\zeta)\right|=1\right\}$. At least one $E_{k}$ is uncountable; suppose $E_{1}$ is. Since $\Gamma_{j}$ is an analytic simple closed curve, there is a real analytic map $\psi$ from the real line onto $\Gamma_{j}$ which is locally a homeomorphism. Then $1-\left|\phi_{1} \circ \psi\right|^{2}$ is real analytic and has uncountably many zeros. Consequently it vanished identically, so $\left|\phi_{1}\right| \equiv 1$ on $\Gamma_{j}$.

Proof of the Theorem. Observe that if $\phi \in \mathcal{O}(\bar{R}),|\phi| \equiv 1$ on $\Gamma_{j}$ and $|\phi|<1$ on $R$, then $d \phi$ is zero free on $\Gamma_{j}$. (See [13, p. 367].)

Let $R_{0}$ be the compact surface from which we obtain $R$, and let $w(P, Q)$ be a Cauchy kernel for $R_{0}$ which is holomorphic on a neighborhood of $\bar{R}$. (See [9, Appendix].) Then if $f \in A(R)$, we can write

$$
f(Q)=\frac{1}{2 \pi i} \int_{\Gamma} f(P) w(P, Q)=\sum_{j=1}^{M} \frac{1}{2 \pi i} \int_{\Gamma_{j}} f(P) w(P, Q) .
$$

Let $f_{j}$ denote the $j$ th summand. It lies in $A(R)$ and is, moreover, holomorphic on a neighborhood of $\Gamma_{k}$ if $k \neq j$. 
We will write $f_{j}=e_{j}+h_{j}$ where $h_{j}$ is holomorphic on a neighborhood of $\bar{R}$ and where $e_{j}$ is of the form $E_{j} \circ \Phi$ for some $E_{j} \in A\left(U^{N}\right)$. Since $h_{j}$ is holomorphic on a neighborhood of $\bar{R}, h_{j}=H_{j} \circ \Phi$ for some $H_{j} \in \mathcal{O}\left(\bar{U}^{N}\right)$ so the proof of the decomposition $f_{j}=e_{j}+h_{j}$ is sufficient to establish the theorem.

Consider $f_{1}$. Let $\psi_{1}$ be one of the $\phi_{k}$ which is identically one in modulus on $\Gamma_{1}$. Thus $\psi_{1}$ maps $\Gamma_{1}$ in a $\mu$-to-one manner onto the unit circle, and since $d \psi_{1}$ has no zeros on $\Gamma_{1}$, there is an annulus $B_{1} \subset R$ one of whose bounding curves is $\Gamma_{1}$, and which is mapped in a $\mu$-to-one fashion by $\psi_{1}$ onto an annulus

$$
B=\{z \in C: 1>|z|>\varepsilon\}
$$

for some $\varepsilon>0$. By the theory of Alling [1], there exist functions $g_{1}, \ldots, g_{\mu}$ holomorphic on a neighborhood of $\bar{B}_{1}$ which constitute a free basis for $A\left(B_{1}\right)$ over $\psi_{1}^{*} A(B)$. Let $f_{1}=\sum_{m=1}^{\mu} g_{m} \tilde{f}_{m}^{(1)} \circ \psi_{1}, \tilde{f}_{m}^{(1)} \in A(B)$.

If $\varepsilon$ is chosen close enough to one, the set $\psi_{1}^{-1}(B)$ will be a union $B_{1} \cup S$ where $\bar{B}_{1} \cap \bar{S}=\varnothing$. Then for a suitable neighborhood $\Omega$ of $\bar{B} \times U^{N-1}, \Phi(V) \cap \Omega$ will be a submanifold of $\Omega$ which decomposes into an annulus containing and only slightly larger than $\Phi\left(B_{1}\right)$, call this piece $M_{1}$, and another piece, $M_{2}$, which contains $\Phi(\bar{S})$. If $\Omega$ is small enough, $\Phi_{1}^{-1}\left(M_{1}\right)$ will lie in the domain of definition of all the $g_{m}$. Let $G_{j} \in \mathcal{O}(\Omega)$ be such that $G_{j} \circ \Phi=g_{j}$ on $\bar{B}_{1}$ and $G_{j} \circ \Phi=0$ on $S$. (The set $S$ may be empty; in this case, we may disregard the second condition imposed on $G_{j}$.)

Define $F_{1}$ on $\bar{B} \times \bar{U}^{N-1}$ by

$$
F_{1}\left(z_{1}, \ldots, z_{N}\right)=\sum G_{m}\left(z_{1}, \ldots, z_{N}\right) \tilde{f}_{m}^{(1)}\left(z_{1}\right)
$$

This function is in $A\left(B \times U^{N-1}\right)$, and for fixed $z_{1} \in \bar{B}$, it is holomorphic in $\left(z_{2}, \ldots, z_{N}\right)$ in a neighborhood of $\bar{U}^{N-1}$. We have the decomposition $F_{1}=F_{1}^{+}-F_{1}^{-}$where

$$
F_{1}^{+}\left(z_{1}, \ldots, z_{N}\right)=\frac{1}{2 \pi i} \int_{|\zeta|=1} \frac{F_{1}\left(\zeta, z_{2}, \ldots, z_{N}\right)}{\zeta-z_{1}} d \zeta
$$

and

$$
F_{1}^{-}\left(z_{1}, \ldots, z_{N}\right)=\frac{1}{2 \pi i} \int_{|\zeta|=8} \frac{F_{1}\left(\zeta, z_{2}, \ldots, z_{N}\right)}{\zeta-z_{1}} d \zeta .
$$

On $B_{1}$, we have $f_{1}=F_{1}^{+} \circ \Phi-F_{1}^{-} \circ \Phi$. Since $F_{1}^{+} \in A\left(U^{N}\right)$, it follows that $F_{1}^{-} \circ \Phi$ continues to an element $h_{1}$ of $A(R)$. We assert that $h_{1}$ is holomorphic on a neighborhood of $\bar{R}$. Consider $\zeta_{0} \in \partial R$. Two cases are possible. It may be that $\left|\psi_{1}\left(\zeta_{0}\right)\right|<1$. Since for each $\zeta$ with $|\zeta|=1, F_{1}\left(\zeta, z_{2}, \ldots, z_{N}\right)$ is holomorphic on a neighborhood of $\bar{U}^{N-1}$, the formula for $F_{1}^{+}$shows it to be holomorphic in a neighborhood of $\Phi\left(\zeta_{0}\right)$. Since $f_{1}$ is holomorphic at $\zeta_{0}$, it follows that $h_{1}$ is necessarily holomorphic there. If $\left|\psi_{1}\left(\zeta_{0}\right)\right|=1$ and $\zeta_{0} \in \bar{B}_{1}$, then $h_{1}(\zeta)=F_{1}^{-}(\Phi(\zeta))$ for $\zeta$ near $\zeta_{0}$, and this is evidently holomorphic near $\zeta_{0}$. If $\left|\psi_{1}\left(\zeta_{0}\right)\right|=1$ and $\zeta_{0} \notin \bar{B}_{1}$, then $f_{1}$ is holomorphic near $\zeta_{0}$ and we have $h_{1}=f_{1}+F_{1}^{+} \circ \Phi$. We have that $F_{1} \circ \Phi \equiv 0$ near $\zeta_{0}$, so it is enough to prove that near $\zeta_{0}, F_{1}^{-} \circ \Phi$ is holomorphic. Again, since for $\zeta$ with $|\zeta|=\varepsilon$, 
$F\left(\zeta, z_{2}, \ldots, a_{N}\right)$ is holomorphic on $\bar{U}^{N-1}$, this is immediate from the formula for $F_{1}^{-}$. This concludes the proof of the theorem.

\title{
REFERENCES
}

1. N. L. Alling, Extensions of meromorphic function rings over non-compact Riemann surfaces. I, Math. Z. 89 (1965), 273-299.

2. E. Bishop, A minimal boundary for function algebras, Pacific J. Math. 9 (1959), 629-642.

3. - Some global problems in the theory of functions of several complex variables, Amer. J. Math. 83 (1961), 479-498.

4. S. Bosch, Endliche analytische Homomorphismen, Nachr. Akad. Wiss. Göttingen Math.-Phys. Kl. 5 (1967).

5. R. Gunning and H. Rossi, Analytic functions of several complex variables, Prentice-Hall, Englewood Cliffs, N. J., 1965.

6. K. Hoffman and H. Rossi, The minimal boundary for an analytic polyhedron, Pacific J. Math. 12 (1962), 1347-1354.

7. K. Hoffman, Banach spaces of analytic functions, Prentice-Hall, Englewood Cliffs, N. J., 1962.

8. W. Hurewicz and H. Wallman, Dimension theory, Princeton Univ. Press, Princeton, N. J., 1948.

9. A. Hurwitz and R. Courant, Funktionentheorie, Springer-Verlag, New York, 1964.

10. B. Malgrange, Lectures on functions of several complex variables, Tata Institute for Fundamental Research, Bombay, 1958.

11. R. Narasimhan, Introduction to the theory of analytic spaces, Lecture Notes in Math. no. 25, Springer-Verlag, New York, 1966.

12. W. Rudin and E. L. Stout, Boundary properties of functions of several complex variables, J. Math. Mech. 14 (1965), 991-1006.

13. E. L. Stout, On some algebras of analytic functions on finite Riemann surfaces, Math. Z. 92 (1966), 366-379.

14. O. Zariski and P. Samuel, Commutative algebra, Vol. I, Princeton Univ. Press, Princeton, N. J., 1958.

15. A. Zygmund, Trigonometric series, 2nd ed., Vol. II, Cambridge Univ. Press, New York, 1959.

16. K. Oka, Sur les fonctions analytiques de plusieurs variables, VIII, Lemme fondamental, J. Math. Soc. Japan 3 (1951), 259-278.

\author{
UNIVERSITY OF WISCONSIN, \\ Madison, Wisconsin \\ YALE UNIVERSITY, \\ New Haven, Connecticut \\ UNIVERSITY OF WASHINGTON, \\ Seattle, Washington
}

